\title{
Revisión de procedimientos metodológicos para la reconstrucción glaciar y el cálculo de la altitud de la línea de equilibrio'
}

\author{
Review of methodological procedures for glacier \\ reconstruction and the calculation of the Equilibrium Line \\ Altitude
}

Néstor Campos² ${ }^{\circledR}$

\begin{abstract}
RESUMEN
La altitud de la línea de equilibrio de los glaciares nos indica en qué punto el glaciar está en equilibrio con el clima, en ese punto la cantidad de masa ganada es equivalente a la que se pierde. Es muy común la utilización de la altitud de la línea de equilibrio glaciar como indicador climático, por ello se hace necesario disponer del estado actual de los glaciares, o de su estado durante la época de cuando se quiere reconstruir el clima. Para obtener estos datos es necesario disponer de la superficie, e idealmente, del volumen de los glaciares. Para obtener estas delimitaciones se propone una metodología de trabajo que empieza por realización de esquemas geomorfológicos con el objetivo de poder deducir los límites alcanzados por el hielo en otras épocas y posteriormente proceder a su reconstrucción y al cálculo de las líneas de equilibrio glaciar. Este estudio presenta el estado del arte de las metodologías y los procedimientos utilizados en las últimas décadas para realizar reconstrucciones glaciares y calcular la altitud de las líneas de equilibrio glaciar.
\end{abstract}

Palabras clave: línea de equilibrio glaciar, reconstrucción glaciar, ELA.

\begin{abstract}
The equilibrium line altitude of the glaciers indicates at what point the glacier is in equilibrium with the climate, at that point the amount of gained mass is equivalent to the lost mass. Is very common the use of the equilibrium line altitude as a climatic indicator, for this reason it is necessary to have the current status of the glaciers, or their state during the glacial phase we want to reconstruct. To obtain these data it is necessary to have the surface and, ideally, the volume of the glaciers. In order to obtain these delimitation a metholodogy is proposed, which consist of carrying out geomorphological sketches, in order to be able to deduce the limits reached by the ice in other periods and, after that, to carry out a reconstruction and calculation of the equilibrium line altitudes. This study presents the state of the art of the methodologies and procedures used over the last decades to realize glacial reconstructions and calculate the equilibrium line altitude of the glaciers.
\end{abstract}

Key words: equilibrium line altitude, glacier modeling, ELA. 


\section{Introducción}

Los glaciares no sólo sirven de indicadores del cambio climático, en algunas áreas, el hielo que almacenan supone prácticamente el $100 \%$ de las reservas hídricas de las que dependen miles de personas para su supervivencia (Campos, 2018). A su vez, también suponen un riesgo debido al calentamiento global observado en las últimas décadas, el cual provoca un retroceso del hielo y, en ocasiones, debido también a la localización de algunos glaciares en pendientes con gran desnivel, existe la posibilidad de avalanchas, lahares y otros peligros relacionados con el rápido deshielo.

La monitorización de los glaciares es de vital importancia debido a varios motivos, entre los más importantes están la estrecha relación que tienen con los cambios en el clima. Como se ha comentado anteriormente, las reservas hídricas de agua dulce son vitales para una gran cantidad de población y ecosistemas cercanos a muchos de ellos, y por último los peligros que entrañan, ya que por ejemplo debido a un rápido deshielo, se podrían producir flujos torrenciales de agua, lodo y rocas. Por todo ello es de gran importancia disponer de un inventario de glaciares, con variaciones a lo largo del tiempo de longitudes, áreas, volúmenes y cambios en su masa.

A finales del siglo XIX ya existían planes de modelización de glaciares para conocer los procesos relacionados con los cambios climáticos, mediante la fundación en 1894 de la Comisión Internacional de Glaciares, con el objetivo de coordinar mundialmente esta monitorización. Se continuó después de 1967 con el Servicio Permanente de la Fluctuaciones Glaciares (PSFG: Permanent Service on the Fluctuation of Glaciers) y tras 1976 con la Secretaría Técnica Temporal para el Inventario Mundial de Glaciares (TTS/WGI: Temporary Technical Secretariat for the World Glacier Inventory). Y en 1986 combinando el PSFG y el TTS/WGI, dando lugar al World Glacier Monitoring Service (WGMS), situado en Suiza, que sigue vigente hoy en día. El WGMS recopila datos de observaciones de cambios de masa, volumen, área y longitud de glaciares a lo largo del tiempo, así como información estadística sobre inventarios de glaciares (sitio web del WGMS, 2018).

Los primeros estudios de balance de masa se remontan a 1874 en el glaciar del Ródano (Rhonegletscher), Suiza. Más tarde, en 1914, se inician mediciones en el glaciar Claridenfirn, también en Suiza, mediciones que se siguen realizando a día de hoy y constituye la serie de datos de balance de masa más longeva del mundo (Rivera et al, 2017). A mitades del siglo XX, en el glaciar sueco de Storglaciären empezaron las series modernas de mediciones anuales continuas más largas en 1945-1946. Estas series fueron seguidas por otras en el glaciar Taky de Alaska, en el glaciar noruego Storbreen y en varios glaciares alpinos, norteamericanos y de otras regiones (Cogley et al., 2010). Entre las series más destacadas se encuentran las de los glaciares alpinos de Sarennes y Saint-Sorlin en Francia y Hintereisferner, Kesselwandferner y Sonnblick en Austria, iniciadas entre 1949 y 1959 (Rivera et al., 2017). En Norteamérica, las más destacadas son las del glaciar South Cascade en Washington, donde se iniciaron series de mediciones en el año 1958 y las del glaciar White en Canadá en el año 1959. Multitud de series se han iniciado desde entonces, la mayoría de ellas pueden encontrarse en el WGMS.

También, aparecieron numerosos documentos analizando la metodología para las mediciones de balance de masa a medida que se unificaban métodos de trabajo de campo y de gabinete. Estos métodos fueron analizados inicialmente por Østrem y Stanley $(1966 ; 1969)$, apareciendo posteriormente trabajos más actualizados (e.g. Østrem y Brugman, 1991) que a parte de la meto- 
dología para la obtención de los datos aportaba información de técnicas de organización en el campo y datos relativos a la instrumentación y otros trabajos (e.g. Kaser et al., 2003; Hubbard y Glasser, 2005) describiendo más generalmente la metodología y técnicas para los trabajos de campo (Cogley et al., 2010).

Desafortunadamente, existen lugares donde no es posible la realización de trabajo de campo debido a la dificultad de acceso al terreno, extensión del área o limitaciones económicas para realizar estos trabajos. En estos casos se puede realizar una reconstrucción glaciar "en gabinete". Para ello existen diversas metodologías de trabajo, dependiendo si se trata de reconstruir glaciares actuales en épocas actuales o pasadas, o glaciares ya extinguidos (paleoglaciares). Estas metodologías también son útiles para apoyar, complementar y analizar la validez de los datos instrumentales obtenidos en trabajos de campo. A mediados del siglo XIX y principios del siglo XX aparecieron estudios pioneros en los que se relacionaban glaciaciones con el clima. Geikie (1909) estudió glaciaciones en los Alpes, destacando los bloques erráticos entre las evidencias más notorias de la acción del hielo en zonas alpinas. También Davis (1909) analizó los Alpes durante el periodo glaciar. Por su parte, Penk (1906) analizó las características climáticas de la Pequeña Edad del Hielo. A partir de entonces y hasta hoy en día, una gran cantidad de estudios analizan la relación de los glaciares con el clima. Los glaciares ofrecen el potencial para reconstruir el clima pasado en escalas que van desde décadas hasta milenios (Mackintosh et al., 2017). Una correcta reconstrucción de los paleoglaciares permitirá reconstruir la paleoELA con exactitud, lo cual ayudará a conocer las condiciones climáticas que existían en el pasado. El objetivo de este estudio es hacer una revisión de los procedimientos metodológicos utilizados para realizar investigaciones sobre reconstrucción glaciar, desde el análisis geomorfológico inicial hasta el cálculo de la altitud de la línea de equilibrio glaciar (término nombrado de aquí en adelante como Equilibrium Line Altitude, por sus siglas en inglés: ELA), pasando por la reconstrucción tridimensional de los glaciares con el objetivo de obtener el volumen de hielo.

\section{Procedimientos metodológicos}

El flujo de procesos general consiste en la realización incial de un esquema o cartografía geomorfológica de los glaciares que queramos reconstruir, posteriormente se procederá a la reconstrucción de los mismos mediante diferentes métodos. Finalmente, se realizará el cálculo de la altitud de la línea de equilibrio glaciar. A continuación, se muestran las metodologías más utilizadas actualmente para la realización de cada uno de estos procesos.

\subsection{Cartografía geomorfológica}

Con el objetivo de representar la distribución de las formas del relieve, la cartografía geomorfológica es uno de los métodos más utilizados, ya que nos permite detectar con facilidad la distribución y el tipo de los elementos cartografiados. Para identificar las formas morfológicas que indican características de glaciares o la presencia antiguamente de glaciares es conveniente la realización de un esquema o mapa geomorfológico. Para las reconstrucciones paleoglaciares se suelen utilizar los cordones morrénicos que indican dónde llegó el hielo en distintas fases. Estas morrenas son clasificadas tradicionalmente de acuerdo con su localización como finales (en la zona de término del glaciar), laterales (a lo largo de los márgenes laterales) o fronto-laterales 
(Barr y Lovell, 2014). También se utilizan los trimlines que nos muestran la altitud alcanzada por el glaciar y otras características morfológicas que nos indican la presencia de hielo en el pasado, principalmente rocas aborregadas, canales de deshielo y llanuras fluvioglaciares.

En cuanto a la leyenda utilizada, por lo general, y haciendo referencia a la investigación actual, en áreas donde existen o han existido glaciares, fecuentemente de alta montaña, se presentan unas formas del relieve más complejas, las cuales requieren un mayor número de símbolos y por consiguiente una leyenda más completa. La escasez de mapas geomorfológicos detallados en áreas de alta montaña, en relación a otras áreas, hace que haya menos tipos de leyendas dedicadas para ello, por lo que en las últimas décadas se han desarrollado nuevas simbologías para este propósito, muchas de ellas ya adaptadas para su uso combinado con sistemas de información geográfica. Dos de las leyendas adecuadas para la cartografía de alta montaña son las de Peña et al. (1997) y la del Instituto de Geografía de la Universidad de Lausana (IGUL, 1996). La leyenda propuesta por Peña et al. (1997) tiene como objetivo principal facilitar un sistema flexible y coherente, con la máxima claridad y facilidad de lectura. Por su parte, uno de los puntos fuertes de la leyenda del IGUL propuesta por (Schoeneich 1993), es que, a parte de ser de fácil comprensión, existe una adaptación (Lambiel et al. 2013) para utilizarla con uno de los softwares más populares de Sistemas de Información Geográfica.

La cartografía geomorfológica es un método tradicional para conseguir el objetivo de representar el relieve de una forma clara y entendible. En primer lugar, se realiza un análisis geomorfológico con el objetivo de identificar elementos del terreno, este análisis se produce mediante campañas de campo (idealmente) y más tarde, ya en gabinete, con el análisis de las fotografías aéreas disponibles, a veces con una fotointerpretación "tradicional" previa de las formas de relieve del área de estudio mediante un estereoscopio y pares de fotogramas de vuelos. Más adelante se digitalizan estas delimitaciones con el objetivo de ser tratadas en un ordenador mediante un Sistema de Información Geográfica o de diseño asistido por ordenador. En otros casos, si no existe la posibilidad de realizar trabajo de campo, las formas del relieve se digitalizan directamente en el ordenador gracias a imágenes satélite de gran calidad.

Más adelante se realiza el diseño del mapa geomorfológico, siguiendo las directrices de la leyenda elegida, la combinación de leyendas o, en caso deseado, una leyenda creada específicamente para el mapa a realizar. Por último, se procede a realizar el diseño final y "acoplamiento" de todos los elementos del mapa utilizando el software de diseño gráfico elegido para obtener la versión final del mapa.

De acuerdo con Chandler et al., (2018), la cartografía se beneficia a menudo de la utilización de un rango de imágenes y resoluciones, permitiendo que las ventajas de cada tipo de datos se integren para producir un mapa geomorfológico preciso. La cartografía geomorfológica ha demostrado ser de gran utilidad como un primer paso para la realización de reconstrucciones glaciares y, como consecuencia de ello, poder posteriormente reconstruir las ELAs.

\subsection{Metodologías de reconstrucción Glaciar}

Conocer el espesor del hielo es de vital importancia para muchos estudios glaciológicos, bien sea en glaciares actuales como en paleoglaciares. Con el objetivo de realizar un cálculo de es- 
pesores del hielo en la superficie de glaciares o paleoglaciares existen diversas metodologías aplicables dependiendo de varias particularidades del caso en concreto, ya bien sea la zona en la que se encuentra, el tipo de glaciar que reconstruir o la cantidad, tipo y series de datos de los que se dispone. La mayoría de estos métodos son válidos únicamente para glaciares de valle (tabla 1), ya que no tienen la complejidad suficiente como para afrontar la modelización de mantos de hielo continentales sin control topográfico. Las diferentes metodologías de reconstrucción más aplicadas a lo largo de los años son las siguientes:

\subsubsection{Schilling y Hollin (1981).}

Este método numérico asume que el hielo tiene una plasticidad perfecta y produce perfiles de hielo teóricos a lo largo del glaciar estudiado. Schilling y Hollin (1981) adaptaron la ecuación de Nye (1952) a los glaciares de valle, incorporando un factor de forma que representa la proporción del esfuerzo conductor del hielo soportado por la superficie topográfica del lecho.

Siguiendo la ecuación del equilibrio mecánico (para un glaciar infinitamente ancho) propuesta por Nye (1952),

$$
\tau=\rho g t \Delta h / \Delta x(1)
$$

Donde $\tau$ es el esfuerzo de cizalla basal, $\rho$ es la densidad del hielo $\left(\sim 900 \mathrm{kgm}^{-3}\right), \mathrm{g}$ la fuerza de la gravedad $\left(981 \mathrm{cms}^{-2}\right), \mathrm{t}$ el espesor del hielo, h es la elevación del hielo respecto a la superficie y x la distancia horizontal desde el frente glaciar. Schilling y Hollin (1981) introdujeron la ecuación siguiente:

$$
h i+1=h i+\left(\frac{\tau Y}{t}\right) i\left(\frac{\Delta x}{\rho g}\right)(2)
$$

Donde $\mathrm{h}$ es la elevación de la superficie del hielo, $\mathrm{t}$ el espesor (calculado como $\mathrm{t}=\mathrm{h}-\mathrm{B}$, siendo $B$ la elevación del lecho), $\tau_{y}$ es el límite plástico y $\Delta x$ es un intervalo específico de distancia a lo largo del eje $\mathrm{x}$.

El factor de forma propuesto por Nye (1952) es un término adimensional incluido con el objetivo de representar la fricción a lo largo del lecho glaciar como una fracción de la resistencia neta del lecho (Jiskoot, 2011), esto es, la proporción de la tensión conductora del hielo soportada por el lecho:

$$
\tau_{B}=\tau_{Y}=c / \tau_{D}(3)
$$

Donde $\tau_{B}$ es el esfuerzo de cizalla basal, $\tau_{D}$ es el esfuerzo conductor y asumiendo que en la línea de flujo $\tau_{\mathrm{B}}=\tau_{\mathrm{D}^{\prime}}$ se define el factor de forma c como

$$
c t=A / p(4)
$$

Donde $\mathrm{A}$ es el área de la sección transversal del glaciar, $\mathrm{t}$ es el espesor del hielo en la línea de flujo y $\mathrm{p}$ es el perímetro transversal de la sección. Este factor de forma se incorpora a la ecuación (16) reemplazando $\tau_{\gamma}$ por $\tau_{\gamma} / c$ y quedaría la siguiente ecuación: 


$$
h i+1=h i+\left(\frac{\tau Y}{t c}\right) i\left(\frac{\Delta x}{\rho g}\right)(5)
$$

De acuerdo con Schilling y Hollin (1981), una tabla de factores de forma fue propuesta posteriormente por Nye (1965) con variaciones del ratio amplitud/espesor para valles con secciones transversales parabólicas, elípticas o rectangulares. Este método fue utilizado por Schilling y Hollin (1981) para la reconstrucción de valles glaciados en California y casquetes glaciares de Patagonia.

\subsubsection{Método ITEM}

El método ITEM propuesto por Farinotti et al., (2009) tiene como objetivo estimar el volumen de hielo y la distribución de los espesores en glaciares alpinos. Este método se basa en la conservación de masa y los principios de la mecánica de fluido del hielo. El método realiza una estimación del volumen de flujo de hielo que pasa a través de unos perfiles establecidos manualmente a lo largo de la línea de flujo (también establecida manualmente), para convertir estos datos en espesores de hielo mediante la aplicación de la ley del flujo de Glen (Glen, 1955). Esta ley describe cómo los cristales individuales de hielo se deforman en respuesta al estrés, considerando el hielo como un fluido visco-elástico no lineal (es decir, que tiene características elásticas y viscosas cuando sufre deformaciones).

El flujo de volumen de hielo a través de cada perfil transversal se calcula integrando el campo del balance de masa en la superficie del área inmediatamente superior correspondiente. De acuerdo con el principio de conservación de masa, la distribución del balance de masa debe equilibrarse con la divergencia del flujo de hielo y el cambio de elevación de la superficie resultante Farinotti et al., (2009).

De acuerdo con Farinotti et al., (2017b) se asume que la divergencia de flujo tiene una dependencia lineal de la elevación, y se prescriben dos gradientes separados para las zonas de acumulación y ablación de los glaciares. Los espesores de hielo obtenidos a lo largo de las líneas de flujo individuales se interpola a lo largo del glaciar, y la pendiente de la superficie local se utiliza para modular localmente el espesor de hielo resultante. Este método fue desarrollado y validado en glaciares alpinos situados en Suiza, para los cuales se conocía previamente parte de la topografía del lecho.

\subsubsection{Benn y Hulton (2010)}

Este método presenta una hoja de cálculo programada para la reconstrucción de perfiles teóricos de hielo para paleoglaciares. Está basado en el método de Schilling y Hollin (1981) y combina la localización de evidencias geomorfológicas para detectar zonas que estuvieron glaciadas con la aplicación de modelos numéricos de cálculo para reconstruir la topografía de los paleoglaciares. Este método automatiza la metodología ideada por Schilling y Hollin (1981) e incluye alguna mejora en el cálculo, como por ejemplo en la ecuación (2), donde $\tau_{y}$ y $t$ son los que son en el momento i, pero no son representativos para el intervalo entre i e i+1, y puede haber una tendencia a que los puntos calculados excedan o subestimen el valor deseado (Benn y Hulton, 2010).

Benn y Hulton (2010) incluyen en el modelo la solución propuesta por Van der Veen (1999), quien solucionó este problema calculando el esfuerzo de cizalla basal y el espesor del hielo tam- 
bién en el paso i+1/2, de esta forma también se calcula en el punto medio entre el intervalo i y el $i+1$, como se muestra en la ecuación siguiente.

$$
\text { h2i+1-hi+1(Bi+Bi+1)+hi(Bi+1-ti)-2 } \Delta x \tau_{\gamma} / \varrho g=0(6)
$$

y calculando h+1 utilizando la solución por factorización de ecuaciones cuadráticas.

Uno de los puntos críticos de la aplicación de este método es la elección del valor de esfuerzo de cizalla basal, ya que los valores deben elegirse de acuerdo a observaciones en valles actualmente glaciados (Vieira, 2008).

\subsubsection{El método GlabTop (Glacier bed Topography)}

Este método propuesto por Linsbauer et al., $(2009,2012)$ para modelar los espesores de hielo y la topografía del lecho en glaciares alpinos mediante el uso de sistemas de información geográfica. GlabTop fue el primer método en proponer la utilización de la relación empírica entre el esfuerzo de cizalla basal promedio $\tau$ y el rango de elevación de glaciares individuales (Haeberli y Hoelzle, 1995), para solucionar el cálculo de espesores del hielo en puntos individuales a lo largo de líneas tributarias digitalizadas manualmente. De acuerdo con Farinotti et al., (2017b) a partir de $\tau$ y la pendiente del rango de elevación $\alpha$, el espesor de hielo se calcula como:

$$
h=\tau /(f \circ g \operatorname{sen} \alpha)(7)
$$

Donde $\mathrm{h}$ es el espesor de hielo, $\mathrm{f}$ el factor de forma, $\mathrm{g}$ la densidad del hielo y $\mathrm{g}$ la fuerza de la gravedad.

La dependencia de la pendiente del rango de elevación $\alpha$ implica que el hielo será delgado donde la superficie del glaciar sea escarpada y será grueso donde la superficie glaciar sea plana. Como resultado se obtiene una distribución de espesores de hielo mediante la interpolación de los valores estimados a lo largo de las líneas tributarias digitalizadas anteriormente.

Este método fue calibrado con información geométrica de paleoglaciares y validado con ecosondas en glaciares actuales. Representa una posible alternativa y un test independiente para aproximaciones basadas en la conservación de masa y el flujo glaciar (Linsbauer et al., 2012). Este método se ha utilizado en diversos estudios, como por ejemplo el cálculo, por los propios autores del método, de los espesores del hielo y lechos glaciares de todos los glaciares suizos.

\subsubsection{HF-Model}

El método HF ideado por Huss y Farinotti (2012) se basa en el método ITEM de Farinotti et al., (2009), desarrollándolo más y haciéndolo aplicable a escala global. Tiene en cuenta factores adicionales como el deslizamiento basal, variaciones longitudinales en el factor de forma de los valles, la ablación frontal y la influencia de la temperatura del hielo y el régimen climático (Farinotti et al., 2017a). Además, evita tener que digitalizar manualmente las líneas de flujo de los glaciares. Todos los cálculos se realizan utilizando bandas de elevación, y la amplitud de la banda de elevación se extrapola considerando la pendiente de la superficie local y la distancia hasta los límites del gla- 
ciar. Para cada banda de elevación de 10 metros tanto la hipsometría glaciar como la pendiente media y la amplitud son evaluadas, y los gradientes del balance de masa son estimados en base a la continentalidad del glaciar, la cual se define a partir de la altitud de la línea de equilibrio local (Farinotti et al., 2017b). Utilizando una forma integrada de la ley del flujo de Glen (Glen, 1955), se deduce el espesor de hielo a partir del volumen de los flujos de hielo a través del glaciar. Huss y Farinotti (2012) evaluaron utilizando este método 171.000 glaciares y casquetes glaciares alrededor del mundo, calculando para cada glaciar, la distribución del espesor y volumen de hielo, la pendiente de la superficie, el factor de forma del valle y el esfuerzo de cizalla basal a lo largo del glaciar, obteniendo una incertidumbre total en las estimaciones del volumen del hielo global de $\pm 12 \%$.

\subsubsection{Gantayat et al., (2014)}

El método propuesto por Gantayat et al., (2014) es una aproximación basada en la velocidad del flujo glaciar. En esta propuesta se realiza una estimación de la distribución de los espesores de hielo utilizando velocidades, pendientes y en una forma integrada de la ley del flujo del hielo de Glen (Glen, 1955). Para el cálculo el método se basa en la ecuación del flujo laminar (e. g. Cuffey y Paterson, 2010) y la ley del flujo de Glen, resolviendo la ecuación siguiente:

$$
\mathrm{Us}=\mathrm{Ub}+\mathrm{H} \frac{2 A}{n+1} \tau_{b}^{n}(8)
$$

donde $u_{s} y u_{b}$ son las velocidades de flujo del hielo (superficial y basal respectivamente), la h el espesor del hielo, A es un parámetro de deslizamiento dependiente de la temperatura, estructura, tamaño de grano y contenido de impurezas y $\tau_{b}$ el esfuerzo de cizalla basal. Sustituyendo en la fórmula (8) el esfuerzo de cizalla basal por:

$$
\tau_{b}=f p g H \sin \alpha(9)
$$

se obtiene finalmente la siguiente ecuación:

$$
\mathrm{Us}=\mathrm{Ub}+\frac{2 A}{n+1}(f p g H \sin \alpha)^{n}(10)
$$

donde se asume que el valor del exponente de la ley del flujo de Glen, n, es igual a 3 (e.g. Gantayat et al., 2014). Calculándose para cada área entre las sucesivas bandas altitudinales. Utilizando este método, Gantayat et al., (2014) realizaron estimaciones del espesor de hielo del glaciar Gangotri, en la India, uno de los glaciares más grandes de la región del Himalaya.

Existe otro método relacionado, conocido como "Gantayat-v2", el cual es una versión modificada del método de Gantayat et al., (2014), en la cual la ecuación (10), en lugar de resolverla para las bandas altitudinales, se resuelve a lo largo de puntos discontinuos de líneas tributarias digitalizadas manualmente, y el espesor de hielo resultante se interpola asumiendo espesores de 0 en los márgenes del glaciar (Farinotti et al., 2017b).

\subsubsection{Método GlabTop2}

El método GlabTop2 (Frey et al., 2014) está basado en el mismo concepto que el explicado en el apartado 2.2.4 (Linsbauer et al., 2009, 2012), en el cual se calcula el espesor del hielo a partir 
de una estimación de la pendiente de la superficie y el esfuerzo de cizalla basal (Farinotti et al., 2017b). En GlabTop2 no es necesario digitalizar manualmente las líneas tributarias gracias a que el cálculo de la pendiente se realiza a partir de la pendiente promedio de todas las celdas dentro de un buffer de elevación predeterminado, y la distribución del espesor del hielo total se calcula interpolando a lo largo de todo el glaciar los valores obtenidos anteriormente y los de los márgenes del glaciar, que se sabe que son 0 (Frey et al., 2014). Frey et al., (2014) utilizó este método para calcular volúmenes de glaciares en la región Himalaya-Karakoram.

\subsubsection{Herramienta GlaRe (Glacier Reconstruction)}

GlaRe (Pellitero et al., 2016) es un conjunto de herramientas para el software ESRI ArcGIS programadas en Python, para reconstruir paleoglaciares. Este método está basado en una solución iterativa para la suposición de una plasticidad perfecta de la reología del hielo, explicada por Benn y Hulton (2010) (apartado 2.2.3).

Pellitero et al., (2016) probaron esta metodología en dos sistemas glaciares actuales en los cuales se conoce la topografía subglaciar, como son el casquete glaciar Folgefonna en Noruega y el glaciar de valle Griessgletscher en los Alpes suizos, obteniendo resultados satisfactorios en ambos casos. También más recientemente este método ha sido aplicado en la Península Ibérica por Palma et al., (2017), Fernandes et al., (2017) y Campos et al., (2019) en Sierra Nevada, los Pirineos Centrales y la Sierra de Gredos respectivamente.

\subsubsection{Herramienta VOLTA (Volume and Topography Automation)}

En el proceso de reconstruir topografías pasadas de glaciares actuales no todas las metodologías son válidas, puesto que hay algunas que necesitan la topografía del lecho del valle actual, como si no existiera el glaciar. Para estos casos es de gran utilidad la herramienta VOLTA (Volume and Topography Automation), ideada por James y Carrivick (2016). Se trata de un script Python para ArcGIS que a partir de la delimitación del glaciar y el modelo digital de elevaciones modeliza el espesor del hielo, el volumen y la topografía del lecho. Está basado en la suposición de una plasticidad perfecta de la reología del hielo y tiene en cuenta un componente de rozamiento de las paredes del valle. Este método permite restar el espesor del hielo modelado del modelo digital de elevaciones actual para así obtener la topografía del valle "libre de hielo" y el volumen del glaciar actual.

\subsubsection{Método GC2D}

Kessler et al. (2006) desarrollaron un modelo numérico que simula la formación y la evolución de los glaciares de valle en una superficie topográfica. Mediante la resolución de ecuaciones de flujo de hielo y conservación de masa, el modelo calcula elevaciones de la superficie del hielo. Este modelo desarrollado integra los glaciares y componentes obtenidos mediante simulaciones climáticas, por lo que tiene capacidad de retroalimentación y de forzamiento climático.

Como resultado se obtiene la modelización de la extensión glaciar sobre una rejilla teniendo en cuenta varios componentes climáticos como son la ELA y el balance de masa. También se pueden incluir otros procesos que modifican la distribución del hielo como pueden ser las avalanchas 
en pendientes con alto grado de inclinación. De acuerdo con Kessler et al. (2006), este modelo permite simular la evolución de glaciares de valle a lo largo de milenios.

Tabla1. Resumen métodos de reconstrucción glaciar

\begin{tabular}{|l|l|c|l|}
\hline Metodología & \multicolumn{1}{|c|}{ Características principales } & Tipos & Referencias \\
\hline $\begin{array}{l}\text { Schilling y } \\
\text { Hollin (1981) }\end{array}$ & $\begin{array}{l}\text { Basado en la plasticidad perfecta } \\
\text { del hielo }\end{array}$ & $\begin{array}{c}\text { Glaciares de } \\
\text { valle }\end{array}$ & Schilling y Hollin (1981) \\
\hline Método ITEM & $\begin{array}{l}\text { Basado en la conservación de masa } \\
\text { y los principios de la mecánica de } \\
\text { fluido del hielo }\end{array}$ & $\begin{array}{l}\text { Glaciares } \\
\text { alpinos }\end{array}$ & Farinotti et al., (2009) \\
\hline $\begin{array}{l}\text { Benn y Hulton } \\
\text { (2010) }\end{array}$ & $\begin{array}{l}\text { Automatización del método de Schi- } \\
\text { lling y Hollin (1981) }\end{array}$ & $\begin{array}{c}\text { Glaciares de } \\
\text { valle }\end{array}$ & Benn y Hulton (2010) \\
\hline $\begin{array}{l}\text { Método } \\
\text { GlabTop }\end{array}$ & $\begin{array}{l}\text { Utilización de la relación empírica } \\
\text { entre el esfuerzo de cizalla basal } \\
\text { promedio y el rango de elevación de } \\
\text { glaciares individuales }\end{array}$ & $\begin{array}{l}\text { Glaciares } \\
\text { alpinos }\end{array}$ & Linsbauer et al., (2009, \\
2012)
\end{tabular}

\subsection{Metodologías de cálculo de la Línea de Equilibrio Glaciar}

Los glaciares suelen dividirse en dos partes principales, la zona de acumulación y la zona de ablación. En la zona de acumulación predominan los procesos de ganancia de masa, como son la precipitación en forma de nieve, la acumulación por viento o avalanchas, etc. y en la zona de ablación sucede lo contrario, se pierde masa debido principalmente al deshielo. El balance de masa glaciar es un concepto utilizado para cuantificar la ganancia o pérdida de hielo de un sistema glaciar, si la acumulación es mayor que la ablación el glaciar tiene un balance de masa positivo. Si la ablación es mayor entonces el glaciar tiene un balance de masa negativo. El tiempo de respuesta glaciar es el tiempo que tarda un glaciar en ajustar su geometría a un nuevo estado estable después de un cambio en el balance de masa causado por una variación del clima. 
El balance de masa de la superficie glaciar es muy sensible a las variaciones climáticas en áreas ubicadas a gran altitud (Kaser et al., 2006; Huss, 2012), viéndose reflejadas estas variaciones en cambios en la acumulación y en la ablación del glaciar (Six y vincent, 2014).

Uno de los parámetros más útiles empleados para cuantificar el efecto del clima en los glaciares y para la caracterización ambiental de los ámbitos glaciados es la ELA. La ELA se define como la altitud teórica que separa la zona de acumulación y ablación de un glaciar, donde la acumulación anual de nieve es igual a la ablación, de forma que el balance de masa es igual a 0 (Porter, 1975a; Meierding, 1982; Dahl y Nesje, 1992; Serrano y González-Trueba, 2004). De acuerdo con Benn et al. (2005) la altitud de la ELA es raramente constante a lo largo de un glaciar, pero varía con patrones de acumulación de nieve, zonas de sombra y otros factores, por lo que se ha utilizado el concepto de ELA estable.

Existen distintos métodos para el cálculo de la ELAs y paleoELAs, los más comunes que han sido aplicados a lo largo de las últimas décadas y que se explican a continuación son los métodos THAR, MGE, CFA, GT, MELM, AA, AABI, AAR, AABR (tabla 2). Siendo más utilizados actualmente los métodos $A A R$ y $A A B R$, quedando los anteriores en desuso o siendo utilizados con mucha menor frecuencia.

\subsubsection{Metodología de cálculo de ELAs mediante el método THAR}

En el método del cálculo de paleoELAs THAR (del inglés toe-to-headwall altitude ratios) se asume que la ELA se encuentra en algún punto fijo de la distancia vertical entre los puntos más bajos del glaciar y los más altos (figura 1). Este punto intermedio varía según el ratio aplicado, siendo un ratio de 0.5 la distancia justo a la mitad entre el frente y la cabecera del glaciar. Varios autores han modificado este ratio para sus cálculos con valores menores a 0.5 obteniendo así una ELA menor que la elevación media (i.e. Meierding, 1982; Klein et al., 1999), o mayores a 0.5 en el caso de cálculo de ELAs en glaciares cubiertos de derrubios (i.e. Clark et al., 1994), debido al efecto que producen los derrubios en el tamaño de las zonas de ablación (Benn y Lehmkuhl, 2000).

Figura No 1. Método THAR (Toe-to-headwall Altidude Ratio) de cálculo de ELAs. Fuente: Elaboración propia basada en Porter (2001).

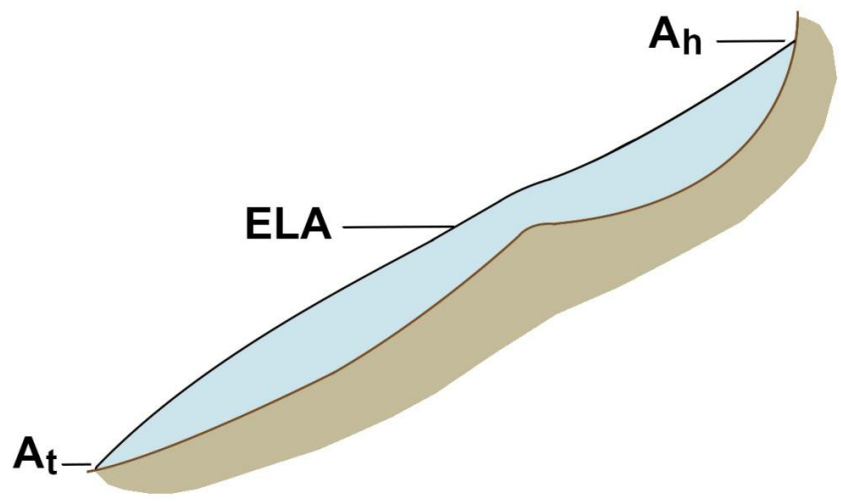


El nivel de la ELA puede estimarse con la ecuación siguiente:

$$
E L A=A t+T H A R(A h-A t)
$$

y por lo tanto:

$$
\text { THAR }=(E L A-A t) /(A h-A t)
$$

Siendo THAR el ratio aplicado, Ah la altitud de la cabecera y At la altitud del frente glaciar.

Las desventajas del método THAR para el cálculo de ELAs son que no tiene en cuenta la hipsometría ni el balance de masa del glaciar.

El método THAR, aunque es más antiguo, fue popularizado por Porter (1981) y Meierding (1982) y fue ampliamente aplicado en las décadas finales del siglo pasado, variando el valor del ratio según distintos autores y zonas de estudio. Meierding (1982) utilizó un ratio de 0.4 en glaciares de Colorado, mientras que valores entre 0.4 y 0.5 fueron utilizados por Porter et al., (1983). Osmaston $(1965,1975)$ propuso ratios de $0.46,0.5$ y 0.57 para distintos grupos de glaciares del valle del Rwenzori (Uganda). Más estudios se han realizado en otras zonas. En los Andes utilizando el método THAR, en la Cordillera Blanca Rodbell (1992), utilizó los ratios 0.2 y 0.4, Fox y Bloom (1994) y Klein et al., (1999) propusieron un ratio de 0.45 en sus estudios en los Andes peruanos, bolivianos y chilenos. Por su parte, Benn et al., (2005) consideraron un ratio de 0.5 como el más adecuado para las latitudes medias y altas. También se aplicó este método en otros tipos de glaciares, Clark et al., (1994) determinaron que para glaciares cubiertos en California el ratio debía de ser 0.6-0.8, más alto debido al efecto de la cubierta de derrubios.

\subsubsection{Metodología de cálculo de ELAs mediante el método MGE}

El método MGE (del inglés Median Glacier Elevation) o también conocido por método Kurowski suscribe que la ELA se encuentra en la altitud media del glaciar. Suponiendo este método (Kurowski, 1891) que para un glaciar que está en equilibrio donde el balance de masa tiene relación lineal con la altitud desde el frente hasta la cabecera, la ELA sería igual a la altitud media del glaciar, asumiendo algunos autores (i.e. Louis, 1955) que existe una relación de proporción entre las áreas de acumulación y de ablación de 1:1. Aunque como apunta Serrano y González-Trueba (2004), según Gross et al., (1977), el método original desarrollado por Kurowski no se corresponde con estas consideraciones, ya que no tiene en cuenta la curva hipsográfica y la topografía del glaciar, parámetros utilizados por Kurowski en su trabajo original para determinar la altitud media del glaciar.

Diversos autores aplicaron este método durante el siglo XX (e.g. Ahlmann, 1948; Meier y Post, 1962; Hoinkes, 1970; Meierding, 1982). De sus resultados se puso de manifiesto que este método tiende a sobreestimar la ELA, puesto que la ablación disminuye con la altura más rápido de lo que aumenta la acumulación, y en el caso de glaciares de valle de latitudes medias, se sitúa por debajo de la altitud media del glaciar (Serrano y González-Trueba, 2004). 


\subsubsection{Metodología de cálculo de ELAs mediante el método CFA}

El método de cálculo de ELAs Altitud del Fondo de Circo Glaciar (conocido en inglés como Cirque Floor Altitudes) propuesto por Péwé y Reger (1972) se basa solamente en la situación del glaciar y tiene como parámetro principal la altitud del fondo del circo glaciar. El método afirma que la ELA de un glaciar confinado a un circo se deduce que no está muy por encima de la altitud media de la base del circo. En el caso de que el glaciar se expanda más allá del umbral del circo la ELA será más baja que el umbral del circo (figura 2).

Figura No 2. Método de cálculo de ELAs CFA (Cirque Floor Altitudes). Fuente: Elaboración propia basada en Porter (2001).

(a)

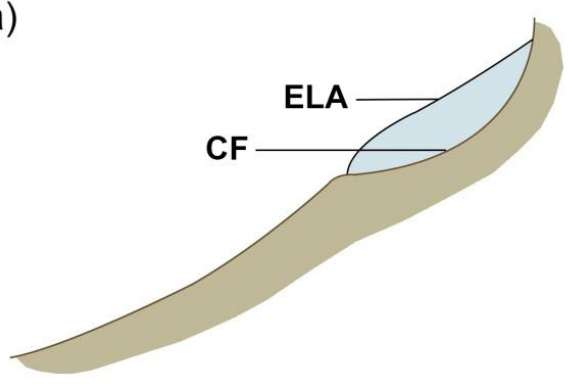

(b)

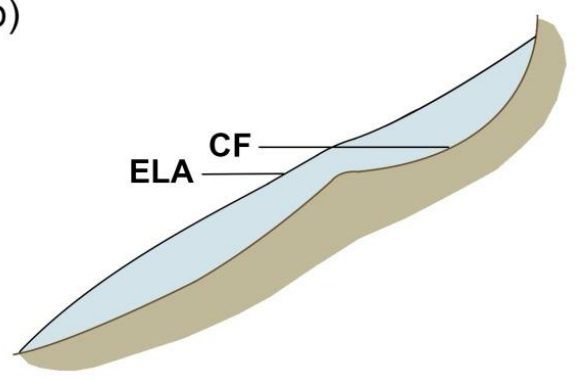

Este método tiene múltiples inconvenientes debido a las limitaciones topográficas y morfológicas, además, sólo es aplicable a tipos específicos de glaciares y tiene un valor muy regional. Este método ha sido utilizado en algunas ocasiones como proxy para el cálculo de paleoELAs (e.g. Péwé y Reger, 1972; Nogami, 1972, 1976; Fox y Bloom, 1994).

\subsubsection{Metodología de cálculo de ELAs mediante el método GT}

EL método del umbral de glaciación (del inglés Glaciation Threshold) se basa en la distribución de los glaciares (Østrem, 1966). Calcula, en un área limitada, la altitud media entre la cima más alta que no está glaciada y la cima glaciada de menor altitud (figura 3).

Figura No 3. Método Glalciation-Threshold de cálculo de ELAs. Fuente: Elaboración propia basada en ilustración de Porter (2001).

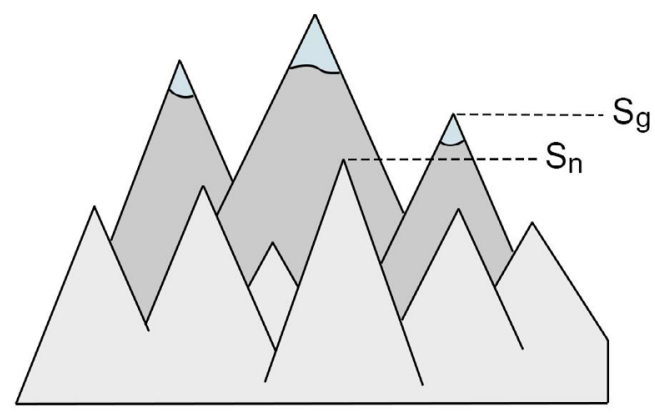


Se calcula mediante la fórmula siguiente:

$$
\text { GT: Sn+0.5 (Sg-Sn) }
$$

siendo Sn la cima no glaciada que se encuentra a más altitud y Sg la cima glaciada con menos altitud.

Este método no puede ser aplicado en montañas aisladas y funciona mejor para estimaciones regionales de la ELA en glaciares actuales, ya que para estimaciones de paleoELA es más difícil saber qué cimas estaban glaciadas y cuales no lo estaban y se añade esa incertidumbre al cálculo.

De acuerdo con Meierding (1982), el método es sencillo de utilizar, por lo que se prepararon mapas de umbrales de glaciación de British Columbia y Alberta (Østrem, 1966), del oeste de Groenlandia (Weidick, 1968), de la Isla Baffin (Andrews y Miller, 1972), de la isla Queen Elizabeth en Canada (Miller et al., 1975) y en los Alpes del sur de Nueva Zelanda (Porter, 1975b). Estos mapas de umbrales de glaciación también se han realizado con el objetivo de reconstruir glaciares del Pleistoceno en Sierra Nevada, California (Wahrhaftig y Birman, 1965), en el norte de Noruega (Anderson, 1968) y en las Cascade Range de Washington (Porter, 1977).

\subsubsection{Metodología de cálculo de ELAs mediante el método MELM}

El método de cálculo de paleoELAs MELM (del inglés Maximum Elevation of Lateral Moraines), propuesto por Lichtenecker (1936) y Visser (1938), en el que se define la ELA a partir de la altitud máxima alcanzada por las morrenas laterales. También conocido como método de Lichtenecker, este método utiliza las bases teóricas de flujo y deposición glaciar, estableciendo que en la parte en que las líneas de flujo convergen hacia el centro por encima de la ELA y divergen hacia el exterior por debajo de la ELA, las morrenas laterales se depositan sólo en la zona de ablación, justo en la zona por debajo de la ELA (Serrano y González-Trueba, 2004). Esto es, la altitud en la que hay un cambio del movimiento del hielo de submergente a emergente, así que la elevación máxima de las morrenas laterales se corresponde con el valor de la ELA en esa fase glaciar (figura 4).

Figura No 4. Método MELM (Maximum Elevation of Lateral Moraines) de cálculo de ELAs. Fuente: Elaboración propia basada en Porter (2001).

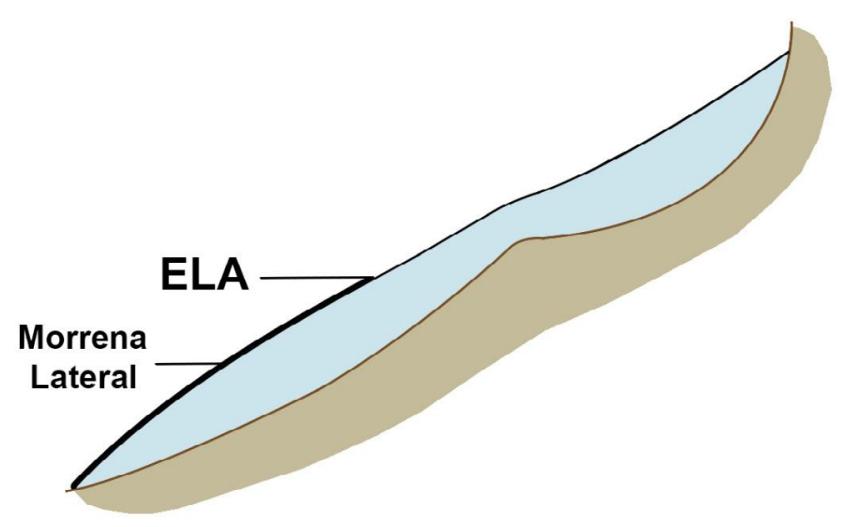


El método MELM para el cálculo de paeloELAs tiene como gran inconveniente la conservación de las morrenas en las que se basa el mismo método, ya que si estas no están bien conservadas o están erosionadas la fiabilidad de los resultados disminuirá y el resultado podrá estar distorsionado.

Meierding (1982) consideró que la altitud de la morrena lateral más alta es uno de los métodos menos fiables para determinar la ELA pleistocénicos tras su aplicación en el Front Range de Colorado. A pesar de ello, este método ha sido utilizado para el cálculo de ELAs en diversas ocasiones, como por ejemplo en los glaciares Pleistocenos en la isla Baffin (Hawkins, 1980) y en el Monte Kenya (Mahaney, 1990). También este método fue utilizado por Bromley et al., (2011), por Úbeda (2011) y por Alcalá (2014) en los Andes Centrales de Perú.

\subsubsection{Metodología de cálculo de ELAs mediante el método AA}

El método AA de cálculo de ELAs (acrónimo de Area x Altitude) tiene en cuenta la hipsometría del glaciar (esto es la distribución detallada del área de la superficie glaciar con respecto de la altitud) con el objetivo de conseguir resultados más fiables.

La estimación del valor de la ELA se calcula probando con un valor determinado como puede ser por ejemplo la altitud media del glaciar, y se multiplican las áreas de las sucesivas bandas altitudinales por el valor medio de la diferencia por encima o por debajo de la ELA de prueba. La suma indica si el valor de la ELA propuesta tiene que aumentar o disminuir, y el cálculo se repite hasta que la suma da 0 como resultado (Osmaston 2005). El método AA asume que el BR (el cociente de los gradientes de acumulación y ablación) es constante y su valor es igual a 1.

Este método fue ideado por Kurowski (1981) con el objetivo de calcular la ELA en glaciares alpinos y adoptado posteriormente por otros autores (i.e. Drygalski y Machatschek 1942) para sus estudios, aunque su aplicación se vio limitada debido a la escasez de mapas topográficos de áreas glaciadas de montaña (Osmaston, 2005). No fue hasta a partir de mediados de los años 60 y gracias al avance de la informática que hizo más fácil su aplicación, cuando se volvió a utilizar este método, aplicándose en montañas africanas, como el monte Rwenzori (Osmaston 1965, 1989a) y el Kilimanjaro (Osmaston 1989b). También Sissons $(1974,1980)$ lo utilizó para realizar estimaciones de las ELA en los montes Grampianos en Escocia y en el Lake District en Inglaterra, en estos estudios, Sissons simplificó el cálculo de la ELA mediante la siguiente ecuación:

$$
E L A=\Sigma Z^{*} A / \Sigma A
$$

donde $Z$ es la altitud media de cada intervalo altitudinal y A es el área de cada banda altitudinal.

De acuerdo con Osmaston (2005), el método AA proporciona una estimación de la ELA más fiable que las estimadas con los métodos THAR o AAR, siempre que el glaciar no sea un glaciar de valle.

En las últimas décadas, el método AA para el cálculo de ELAs ha sido utilizado en los Andes Centrales de Perú por Úbeda et al. (2006), por Úbeda (2007; 2011) y por Alcalá (2014). 


\subsubsection{Metodología de cálculo de ELAs mediante el método $A A B I$}

El método $A A B I$ (del inglés Area x Altitude Balance Index) de cálculo de ELAs, al igual que los métodos $A A$ y $A A B R$ tiene en cuenta la hipsometría del glaciar. Este método permite la aplicación de cualquier curva de balance de masa en cualquier glaciar cuyas áreas de bandas altitudinales puedan ser calculadas (Osmaston 2005). El BI (Balance Index) es un número que representa el valor relativo del balance de masa en cada altitud. Este método de cálculo de ELAs es similar al método AABR en el cual se testea el balance glaciar para sucesivos valores de prueba de las ELAs. Para cada rango de altitudes, el producto de su área y la diferencia de la altitud con el valor de la ELA de prueba se multiplica por el BI de una tabla de consulta (de la que previamente se conoce la certeza de sus valores). Estos son sumados para estimar el balance para el glaciar completo. Cuando se llegue el punto en el que dos valores de prueba consecutivos de la ELA den como resultado uno positivo y otro negativo, entonces la ELA se encuentra entre esos valores, a una altitud intermedia determinada por el ratio de los dos balances (Osmaston 2005).

\subsubsection{Metodología de cálculo de ELAs mediante el método AAR}

El método AAR (del inglés Accumulation-Area Ratio), también conocido como método Brückner- Richter debido a que tiene su origen en los estudios de estos dos investigadores en los Alpes, a finales del siglo XIX (Brückner 1886, 1887, 1906; Richter 1885, 1888).

La utilización de este método de estimación de la ELA se basa en la asunción de que, bajo condiciones de equilibrio, el ratio entre las áreas de acumulación y de ablación es constante (figura 5).

Figura No 5. Método de cálculo de ELAS AAR. Fuente: modificado de Porter (2001).

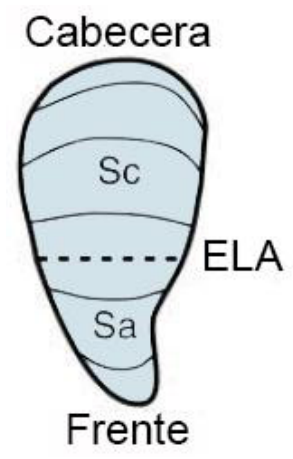

El ratio acumulación-ablación de un glaciar es el ratio del área de acumulación dividido por la suma de sus áreas de acumulación y ablación (figura 5)

$$
A A R=\frac{S c}{S c+S a}(15)
$$

Donde Sc es el área de acumulación y Sa el área de ablación

De acuerdo con Serrano y González-Trueba (2004), inicialmente el método original asumía unas condiciones teóricamente estables, debido a que, en ese momento, no se disponía de los 
datos cuantitativos necesarios para la elaboración de un método de tipo inductivo. Inicialmente se asumió (i.e. Brückner 1886, 1887, 1906) que el límite entre el área de acumulación y el área de ablación tiene una proporción de 3:1 (AAR=0.75), siendo la línea que separa las áreas la línea de equilibrio glaciar. Estudios posteriores (i.e. Drygalski y Machatschek 1942; Meier y Post, 1962; Gross et al., 1977) revisaron esta relación considerando que se subestimaba la ELA y sugirieron la relación 2:1 (AAR=0.67), lo que significaría esto que la superficie de acumulación correspondería a dos tercios de la superficie total del glaciar.

A lo largo del tiempo y del contexto, los valores del ratio han ido variando según los autores que realizaban las estimaciones, Meier y Post (1962) sugirieron que estudios empíricos en glaciares modernos mostraron que, bajo condiciones de equilibrio, el ratio AAR se encontraba mayormente entre 0.5 y 0.8 (i.e., $0.65+-0.15$ ), mientras que glaciares por debajo de 0.5 indicaban balance de masa negativo y por encima de 0.8 regímenes con balance de masa positivos (Kaser y Kerschner, 1994; Serrano y Gomez-Trueba, 2004). Varios experimentos (Meierding 1962; Hawkins, 1980) utilizando un AAR de 0.65 mostraron que ese ratio tenía el error cuadrático medio (RMSE) más bajo, lo que apoyaba la utilización de estos valores en trabajos anteriores.

Para reconstrucciones paleoglaciares, se cree que el rango adecuado está también entre 0.5 y 0.8 , siendo 0.6 característico de glaciares de valle o de circo (Porter, 1977; Benn y Evans, 1998; Nesje y Dahl, 2000; Rea, 2009). Actualmente, el cálculo de ELAs considerando un AAR de 0,6 \pm 0,05 ha aportado los valores más aproximados (figura 6), siendo éste método uno de los más utilizados en la actualidad tanto para glaciares hoy desaparecidos, como para aquellos aparatos actuales en los que se desconozca su balance de masa específico (Serrano y González-Trueba, 2004).

Para calcular la altitud de la línea de equilibrio glaciar por el método AAR, y de acuerdo con Porter (2001), se estima una ELA inicial, si no se tiene una topografía del glaciar se dibujan las curvas de nivel, consistentes con los principios del flujo glaciar (las curvas de nivel en un glaciar en estado de equilibrio son concavas hacia la cabecera del glaciar en el área de acumulación y convexas en el área de ablación, con el grado de concavidad o convexidad incrementándose conforme se alejan las curvas de nivel de la línea de equilibrio). El área entre cada par de curvas de nivel sucesivas se utiliza para generar una curva que muestra la distribución el área acumulada en cada intervalo de altitud.

Figura No 6. Relación entre altitud y acumulación, con un valor de AAR de 0.65. Fuente: Porter (2001).

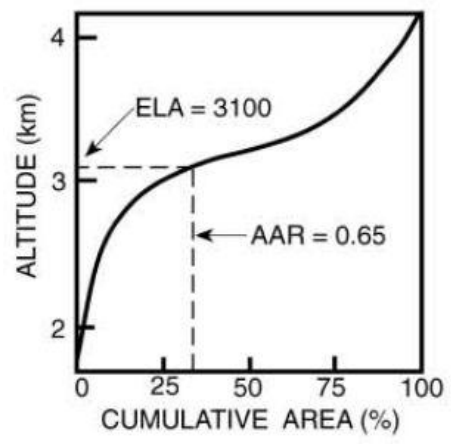


El mayor problema de este método de cálculo de ELAs es que no tiene en cuenta la hipsometría del glaciar, esto es la distribución del área con respecto a la altitud (Osmaston 2005), lo que bajo ciertas circunstancias puede afectar significativamente al resultado obtenido en el cálculo de las ELAs (Mercer, 1961; Furbish y Andrews, 1984; Benn y Evans, 1998: Rea y Evans, 2007, Rea, 2009). Otro problema de este método tiene relación con la precisión de la reconstrucción de la masa glaciar en reconstrucciones paleoglaciares, utilizando para ello huellas morfológicas glaciares heredadas. Las mayores dificultades se plantean a la hora de estimar la extensión y máxima altitud ocupada por el glaciar a través de una estimación del espesor del hielo en la cabecera (Serrano y González-Trueba 2004).

A pesar de estas limitaciones, el método AAR ha sido ampliamente utilizado a lo largo de las últimas décadas para calcular las ELAs en distintas áreas del planeta. Braithwaite y Müller (1980) analizaron con este método glaciares del Ártico, de Norteamérica, de Escandinavia y de Asia Central. En zonas tropicales se han obtenido ELAs en Hawaii (Porter, 1979; Dorn et al., 1991), en México (White y Valastro, 1984; White, 1981), también en numerosas ocasiones se ha utilizado el método AAR para el cálculo de ELAs en la región de los Andes Centrales (e.g. Úbeda, 2011; Alcalá, 2014). En Escocia fue utilizado por Benn y Ballantyne (2005), en Noruega por Lukas (2007). En el sur de Europa, ha sido utilizado en la Península Ibérica (Vieira 2008; Carrasco et al. 2013; López-Moreno et al. 2016, entre otros). Además, Dyurgerov et al, (2009) analizaron el método en 86 glaciares repartidos por todo el mundo.

\subsubsection{Metodología de cálculo de ELAs mediante el método AABR}

EL método de cálculo de ELAs AABR (del inglés Area -Altitude Balance Ratio) se basa, al igual que el método $A A$, en el principio de ponderar el balance de masa en áreas muy por encima $o$ muy por debajo de la ELA en mayor medida que en las áreas cercanas a la ELA (Osmaston 2005) y es más robusto que el método AAR porque tiene en cuenta la hipsometría del glaciar. En este método el resultado se redefine mediante diferentes gradientes lineales del balance de masa.

Este método fue inicialmente propuesto por Osmaston (1975) con el nombre de Area-HeighAccumulation, aunque años más tarde Furbish y Andrews (1984) introdujeron el término Balance Ratio, el cual deriva del cociente de los gradientes de equilibrio de las zonas de ablación y de acumulación (Ramage et al., 2005; Alcalá 2014).

El Balance Ratio se puede obtener a partir de la hipsometría del glaciar mediante la siguiente fórmula (Furbish y Andrews, 1984):

$$
\frac{b n b}{b n c}=\frac{Z c A c}{Z b A b}(16)
$$

o equivalentemente

$$
\frac{b n b}{b n c}=\frac{m c}{m b}
$$

Donde $\left[b_{n b} / b_{n c}\right.$ es el BR], Ac es el área de acumulación, Zc es la altitud media ponderada del área de acumulación, $\mathrm{Ab}$ es el área de ablación y $\mathrm{Zb}$ es la altitud media ponderada del área de ablación. 
Para la estimación de la ELA mediante este método, se selecciona una ELA inicial en base a la curva hipsométrica, luego se compara el BR con la relación de la altura promedio del área de acumulación dividida por la altura promedio del área de ablación. Si el BR es igual a esta relación, el valor de la ELA está ajustado correctamente, de lo contrario hay que revisar el valor de la ELA para encontrar un mejor ajuste (Ramage et al., 2005).

Para el cálculo de paleoELAs, y de acuerdo con Furbish y Andrews (1984), el método AABR se basa en tres suposiciones (Rea, 2009):

1. los gradientes de acumulación y ablación son aproximadamente lineales.

2. el ratio neto entre ablación y acumulación es conocido y permanece fijo en el tiempo.

3. en el caso de los glaciares de valle y de los icefields de meseta cuyos outlet fluían por valles, la topografía mantiene al glaciar "acotado", por lo que un cambio en el clima (y por tanto en el balance de masa) se verá reflejado en un cambio en el frente glaciar.

Para calcular paleoELAs en glaciares extintos mediante el método AABR se puede hacer de distintas formas, si existe un glaciar actual se puede calcular su BR y luego, basados en la suposición anteriormente comentada de que el ratio neto entre ablación y acumulación permanece en el tiempo, se aplica a la hipsometría del paleoglaciar reconstruido para calcular la paleoELA. Esto puede hacerse siempre que se tengan sospechas de que el glaciar actual y el paleoglaciar tengan similares características (Rea y Evans, 2007). Otra alternativa sería estimar el BR de un glaciar cercano y asumir que el glaciar objeto de estudio se encuentra bajo influencia de las mismas condiciones climáticas y glaciológicas. A menudo no existe ni glaciar actual ni glaciares cercanos al área de estudio, en estos casos habría que elegir un BR "respresentativo" (Rea, 2009).

De acuerdo con Benn y Lehmkuhl (2000) los BRs varían con el clima regional, son más bajos en regiones polares donde la ablación depende poco de la altitud y más altos en zonas tropicales donde la ablación sucede durante todo el año. Los glaciares con un BR alto tienen áreas de ablación más pequeñas en relación con el área total del glaciar, debido a que sólo se necesita un área pequeña de ablación para equilibrar las ganancias en la zona de acumulación. En los glaciares con BRs bajos sucede todo lo contrario, estos glaciares requieren grandes áreas de ablación para compensar las ganancias de la zona de acumulación. Por lo tanto, los glaciares tropicales tienden a tener áreas de ablación más pequeñas que los glaciares de latitudes medias (con geometría similar), y su ELA estará más cercana al frente glaciar (Benn y Gemmell, 1997).

Según Rea (2009) un BR de 1 indica gradientes de acumulación y ablación equivalentes, lo que es más probable que ocurra en regiones polares. En un estudio de 22 glaciares de Alaska Furbish y Andrews (1984) estimaron que los BRs eran de aproximadamente 1.8. BRs de 1-8 a 2.2 se han propuesto como representativos de glaciares de latitudes medias con influencia marítima, debido a la alta ablación durante el verano (Benn y Gemmell, 1997; Benn y Evans, 1998; Benn y Lehmkuhl, 2000; Rea, 2009). Se asumen BRs altos (>2.2) como representativos para glaciares tropicales, debido a las altas tasas de ablación durante todo el año. En estudios recientes, Rea (2009) estimó para un conjunto global de glaciares unos BRs "representativos" según tipos de glaciares, basándose en datos de balance de masa recogidos durante 47 años: un AABR global de 1.75+0.71, de latitudes medias con influencia marítima 1.9+-0.81, altas latitudes 2.24+-0.85, América del norte-Costa Oeste 2.09+-0.93, América del norte Rocosas del Este 1.11+-0.1, ártico canadiense 
2.91+-0.35, Svalbard 2.13+-0.52, Oeste de noruega 1.5+-0.4, Alpes europeos 1.59+-0.6, Asia central $1.75+-0.56$ y Kamchatka 3.18+-0.16.

En el modelo sugerido por Osmaston (2005), para grupos de glaciares que se encuentran en la misma zona se sugiere calcular la ELA con un rango de BRs (e.g. 1.0-3.5) y luego elegir el BR con la menor desviación típica, la cual indicará el valor de la ELA con la mayor probabilidad estadística de ser correcta.

Hoy en día, el método AABR es, junto con el AAR, el más utilizado para calcular ELAs. Además de los estudios comentados anteriormente, también ha tenido gran aplicación en los Andes (e. g. Ramage et al., 2005; Forget et al. 2008; Úbeda 2011; Alcalá 2014; Campos 2015) y en el sur de Europa, donde ha sido utilizado ampliamente utilizado en la Península Ibérica (e.g. Vieira 2008; Carrasco et al. 2013, entre otros).

Tabla 2. Resumen métodos cálculo de la ELA

\begin{tabular}{|c|c|c|c|}
\hline Metodología & Cálculo ELA & Características & Referencias \\
\hline $\begin{array}{l}\text { THAR (toe-to-head- } \\
\text { wall altitude ratios) }\end{array}$ & $\begin{array}{l}\text { La ELA se encuentra en algún } \\
\text { punto fijo de la distancia verti- } \\
\text { cal entre los puntos más bajos } \\
\text { del glaciar y los más altos }\end{array}$ & $\begin{array}{l}\text { No tiene en cuenta la hip- } \\
\text { sometría ni el balance de } \\
\text { masa del glaciar }\end{array}$ & $\begin{array}{l}\text { Porter (1981); } \\
\text { Meierding } \\
\text { (1982) }\end{array}$ \\
\hline $\begin{array}{l}\text { MGE (median gla- } \\
\text { cier elevation) }\end{array}$ & $\begin{array}{l}\text { La ELA se encuentra en la alti- } \\
\text { tud media del glaciar }\end{array}$ & $\begin{array}{l}\text { Se calcula la ELA a través } \\
\text { de la curva hipsográfica }\end{array}$ & (Kurowski, 1891) \\
\hline $\begin{array}{l}\text { CFA (cirque floor } \\
\text { altitude) }\end{array}$ & $\begin{array}{l}\text { La ELA en un glaciar de circo } \\
\text { no está muy por encima de la } \\
\text { altitud media de la base del } \\
\text { circo. Si el glaciar se expande } \\
\text { más allá del umbral del circo la } \\
\text { ELA será más baja que el um- } \\
\text { bral del circo }\end{array}$ & $\begin{array}{l}\text { Método con inconve- } \\
\text { nientes debido a las limi- } \\
\text { taciones topográficas y } \\
\text { morfológicas }\end{array}$ & $\begin{array}{l}\text { Péwé y Reger } \\
\text { (1972) }\end{array}$ \\
\hline $\begin{array}{l}\text { GT (glaciation } \\
\text { threshold) }\end{array}$ & $\begin{array}{l}\text { Se basa en la altitud media } \\
\text { entre la cima más alta no gla- } \\
\text { ciada y la cima glaciada con } \\
\text { menor altitud }\end{array}$ & $\begin{array}{l}\text { No puede aplicarse en } \\
\text { montañas aisladas y fun- } \\
\text { ciona mejor para esti- } \\
\text { maciones en glaciares } \\
\text { actuales }\end{array}$ & Østrem, (1966) \\
\hline $\begin{array}{l}\text { MELM (maximum } \\
\text { elevation of lateral } \\
\text { moraines) }\end{array}$ & $\begin{array}{l}\text { Define la ELA a partir de la alti- } \\
\text { tud máxima alcanzada por las } \\
\text { morrenas laterales }\end{array}$ & $\begin{array}{l}\text { Inconveniente para el } \\
\text { cálculo de paleoELAs } \\
\text { por la dependencia de la } \\
\text { buena conservación de } \\
\text { las morrenas }\end{array}$ & $\begin{array}{l}\text { Lichtenecker } \\
\text { (1936); Visser } \\
\text { (1938) }\end{array}$ \\
\hline AA (area $x$ altitude) & $\begin{array}{l}\text { La ELA se calcula teniendo en } \\
\text { cuenta la distribución deta- } \\
\text { llada del área de la superficie } \\
\text { glaciar con respecto de la al- } \\
\text { titud }\end{array}$ & $\begin{array}{l}\text { Este método es igual al } \\
A A B R \text { con } B R=1\end{array}$ & (Kurowski, 1891) \\
\hline
\end{tabular}




\begin{tabular}{|l|l|l|c|}
\hline \multicolumn{1}{|c|}{ Metodología } & \multicolumn{1}{|c|}{ Cálculo ELA } & Características & Referencias \\
\hline $\begin{array}{l}\text { AABI (area x altitu- } \\
\text { de balance index) }\end{array}$ & $\begin{array}{l}\text { Se aplica cualquier curva de ba- } \\
\text { lance de masa en cualquier gla- } \\
\text { ciar cuyas áreas de bandas alti- } \\
\text { tudinales puedan ser calculadas }\end{array}$ & $\begin{array}{l}\text { Tiene en cuenta la hipso- } \\
\text { metría del glaciar }\end{array}$ & $\begin{array}{c}\text { Osmaston } \\
(2005)\end{array}$ \\
\hline $\begin{array}{l}\text { AAR (accumula- } \\
\text { tion-area ratio) }\end{array}$ & $\begin{array}{l}\text { El cálculo de la ELA se basa en } \\
\text { la asunción de que el ratio en- } \\
\text { tre las áreas de acumulación y } \\
\text { de ablación es constante bajo } \\
\text { condiciones de equilibrio }\end{array}$ & $\begin{array}{l}\text { No tiene en cuenta la hip- } \\
\text { sometría del glaciar }\end{array}$ & $\begin{array}{c}\text { Brückner (1886; } \\
1887 ; 1906) ; \\
\text { Richter (1885; } \\
1888)\end{array}$ \\
\hline $\begin{array}{l}\text { AABR (area-altitu- } \\
\text { de balance ratio) }\end{array}$ & $\begin{array}{l}\text { El cálculo de la ELA se basa } \\
\text { en el principio de ponderar el } \\
\text { balance de masa en áreas muy } \\
\text { por encima o muy por debajo } \\
\text { de la ELA en mayor medida que } \\
\text { en las áreas cercanas a la ELA }\end{array}$ & Tiene en cuenta la hipso- \\
metría del glaciar & $\begin{array}{c}\text { Osmaston } \\
(1975) ; \text { Furbish y } \\
\text { Andrews (1984) }\end{array}$ & \\
\hline
\end{tabular}

\section{Conclusiones}

La monitorización de los glaciares es de vital importancia debido a que son sensibles a cambios en el clima, y por tanto el análisis de las tendencias de los glaciares supone una gran ayuda para analizar el cambio climático actual. Gracias al estudio de la evolución de la ELA y del área ocupada por los glaciares es posible conocer el impacto del cambio climático en la actualidad. También se pueden reconstruir condiciones paleoclimáticas basándonos en valores calculados de paleoELAs que condicionaron la extensión de estos glaciares en el pasado.

Para reconstruir la dinámica de los glaciares en el pasado se pueden seguir una serie de procedimientos combinando las distintas técnicas mencionadas a lo largo del presente estudio que han demostrado ser eficientes para este propósito. Los análisis geomorfológicos permiten conocer los límites alcanzados por los glaciares en el pasado. A partir de los límites obtenidos del análisis geomorfológico se realiza la reconstrucción del volumen glaciar mediante modelos numéricos de cálculo. Una vez reconstruido el aparato glaciar, se procede al cálculo de ELAs y paleoELAs mediante el análisis de la masa de hielo reconstruida. A partir de los valores obtenidos se podrán deducir las condiciones paleoclimáticas y la evolución de la dinámica glaciar.

\section{Referencias}

Ahlmann, H. W. (1948). Glaciological research on the North Atlantic coasts. Royal Geographical Society. (Research series 1), London (83 pp).

Alcalá, J. (2014). La evolución volcánica, glaciar y periglaciar del complejo volcánico Ampato (sur de Perú). Tesis doctoral, e-prints Universidad Complutense de Madrid. http:// eprints.ucm. es/29492/ 
Anderson, B. C. (1968). Glacial geology of western Troms, north Norway. Norges Geologiske Undersokelse No. 256.

Andrews, J. T. \& Miller, G. H. (1972). Quatemary history of northern Cumberland Peninsula, Bafftn Island, N.W.T., Canada. Part IV: Maps of the present glaciation limits and lowest equilibrium line altitude for south Baftin Island. Arctic and Alpine Research 4, 45-59.

Barr, I. D. \& Lovell, H. (2014). A review of topographic controls on moraine distribution. Geomorphology, 226, 44-64. http://dx.doi.org/10.1016/j.geomorph.2014.07.030

Benn, D.I. \& Gemmel, A.M.D. (1997). Calculating equilibrium-line altitudes of former glaciers by the balance ratio method: a new computer spreadsheet. Glacial Geol. Geomorphol., 7.

Benn D.I. \& Evans D.J.A. (1998). Glaciers and glaciation. Arnold, London, 734 pp.

Benn, D. \& Lehmkuhl, F. (2000). Mass balance and equilibrium-line altitudes of glaciers in high-mountain environments. Quaternary International, 65-66: 15-29.

Benn, D. I. \& Ballantyne, C. K. 2005. Palaeoclimatic reconstruction from Loch Lomond Readvance glaciers in the West Drumochter Hills, Scotland. J. Quaternary Sci., Vol. 20 pp. 577-592. ISSN 0267-8179

Benn, D. I., Owen, L. A., Osmaston, H. A., Seltzer, G. O., Porterd, S. C. \& Mark, B. (2005). Reconstruction of equilibrium line altitudes for tropical and subtropical glaciers. Quaternary International, 138-139: 8-21. http://dx.doi.org/ 10.1016/j.quaint.2005.02.003

Benn, D.I. \& Hulton, N.R.J. (2010). An Excel spreadsheet program for reconstructing the surface profile of former mountain glaciers and ice caps. Comput Geosci. 36:605-610. doi:10.1016/j.cageo.2009.09.016.

Braithwaite, R.J. \& Müller, F. (1980). On the parameterization of glacier equilibrium line altitude. In: Proceedings of the Riederalp Workshop, 1978. World Glacier Inventory, pp. 263-271

Bromley, R. M., Hall, B. L., Rademaker, K. M., Todd, C. E. \& Racoviteanu, A. E. (2011). Late Pleistocene snowline fluctuations at Nevado Coropuna $\left(15^{\circ} \mathrm{S}\right)$, southern Peruvian Andes. Journal of Quaternary Science, 26: 305-317.

Brückner, E. (1886). Die Hohern Tauern und ihre Eisbedeckung. Zeitschrift des Deutsch-Österreichische Alpenvereins, 17: 163-187, Innsbruck.

Brückner, E. (1887). Die Höhe der Schneelinie und ihre Bestimmung. Meteorologische Zeitschrift, 4: 31-32.

Brückner, E. (1906). Die Höhe der Firnlinie am Hüfigletscher und die Methode der Bestimmung der Höhe der Firnlinie im allgemeinen. Vierteljahrsschrift d. Naturf. Ges. Zürich, 51: 50-54, Zürich. 
Campos, N. (2015). Equilibrium Line Altitude Fluctuation on the South West Slope of Nevado Coropuna Since the Last Glacial Maximum (Cordillera Ampato, Perú). Pirineos, 170, e015. doi: http:// dx.doi. org/10.3989/Pirineos.2015.170008

Campos, N. (2018). Evolución reciente de la criosfera en áreas de alta montaña y su relación con el cambio climático. Tesis doctoral. Universidad Complutense de Madrid.

Campos, N., Palacios, D. \& Tanarro, L. (2019) Glacier reconstruction of La Covacha Massif in Sierra de Gredos (central Spain) during the Last Glacial Maximum. Journal of Mountain Science 16(). https://doi.org/10.1007/10.1007/s11629-019-5382-2

Carrasco, R., de Pedraza, J., Domínguez-Villar, D., Villa, J. \& Willenbring, J. (2013). The plateau glacier in the Sierra de Béjar (Iberian Central System) during its maximum extent. Reconstruction and chronology. Geomorphology. 196. 83-93. 10.1016/j.geomorph.2012.03.019.

Chandler, B. M., Lovell, H., Boston, C. M., Lukas, S., Barr, I. D., Benediktsson, İ. Ö., ... \& Ewertowski, M. W. (2018). Glacial geomorphological mapping: A review of approaches and frameworks for best practice. Earth-Science Reviews, 185, 806-846. https://doi.org/10.1016/j.earscirev.2018.07.015

Clark, D.H., Clark, M.M. \& Gillespie, A.R. (1994). Debris-covered glaciers in the Sierra Nevada, California, and their implications for snowline reconstructions. Quaternary Research 41, 139-153

Cogley, J.G., Hock, R., Rasmussen, L.A., Arendt, A.A., Bauder, A., Braithwaite, R.J., Jansson, P., Kaser, G., Möller, M., Nicholson, L. \& Zemp, M. (2010). Glossary of Glacier Mass Balance and Related Terms. IHP-VII Technical Documents in Hydrology No. 86, IACS Contribution No. 2, UNESCO-IHP, Paris. 114 pp.

Cuffey, K. \& Paterson, W. (2010). The Physics of Glaciers. Elsevier, Oxford, U.K., fourth edition. 715 pp.

Dahl, S.O. \& Nesje, A. (1992). Paleoclimatic based on equilibrium line altitude depressions of reconstructed Younger Dryas and Holocene cirque in Inner Nordfjord, western Norway. Paleogeography, Palaeoclimatology and Palaeoecology, 94: 87-97.

Davis, W. M. (1909). The Alps in the Glacial Period. The Geographical Journal, 34(6), 650-659. https://doi.org/10.2307/1777951

Dorn, R.I., Phillips, F.M., Zreda, M.G., Wolfe, E.W., Jull, A.J.T., Donahue, D.J., Kubik, P.W. \& Sharma, P. (1991). Glacial chronology of Mauna Kea, Hawaii, as constrained by surface-exposure dating. National Geographic Research and Exploration 7, 456-471.

Drygalski, E.V. \& Machatschek, F. (1942). Gletscherkunde, Enzyklopädie der Erdkunde, 261 pg., Wien.

Dumitrashko, N. V. \& Scholz, E. (1978). Classification of geomorphological maps according to scale. In Demek, J. \& Embleton, C: (Eds.): Guide to Medium-Scale Geomorphological Mapping, p. 40. Czechoslovak Academy of Sciences. Akademia. Praga. 
Dyurgerov, M., Meier, M. F. \& Bahr., D. B. (2009). A new index of glacier area change: a tool for glacier monitoring. Journal of Glaciology, Vol. 55, No. 192.

Farinotti, D., Huss, M., Bauder, A., Funk, M., \& Truffer, M. (2009). A method to estimate ice volume and ice thickness distribution of alpine glaciers. Journal of Glaciology, 55(191):422-430. doi: 10.3189/002214309788816759.

Farinotti, D., Brinkerhoff, D. J., Clarke, G. K. C., Fürst, J. J., Frey, H., Gantayat, P., Gillet-Chaulet, F., Girard, C.... Andreassen, L. M. (2017a). How accurate are estimates of glacier ice thickness? Results from ITMIX, the Ice Thickness Models Intercomparison eXperiment, The Cryosphere, 11, 949-970, https://doi.org/10.5194/tc-11-949-2017, 2017.

Farinotti, D., Brinkerhoff, D. J., Clarke, G. K. C., Fürst, J. J., Frey, H., Gantayat, P., Gillet-Chaulet, F., Girard, C.... Andreassen, L. M. (2017b). How accurate are estimates of glacier ice thickness? Results from ITMIX, the Ice Thickness Models Intercomparison eXperiment, Supplementary material. The Cryosphere, 11, 949-970, https:// doi:10.5194/tc-11-949-2017-supplement.

Fernandes, M., Oliva, M., Palma, P., Ruiz-Fernández, J. \& Lopes, L. (2017). Glacial stages and post-glacial environmental evolution in the Upper Garonne valley, Central Pyrenees. Sci Total Environ. 584-585:1282-1299. doi:10.1016/j.scitotenv.2017.01.209.

Forget, M., Thouret, J. C., Kuentz, A. \& Fontugne, M. (2008). Héritages glaciaires, périglaciaires et évolution récente: le cas du Nevado Coropuna (Andes centrales, sud du Pérou). Géomorphologie: relief, processus, environnement, 2: 113-132.

Fox, A. N. \& Bloom, A. L. (1994). Snowline altitude and climate in the Peruvian Andes (50-170 S) at present and during the Latest Pleistocene Glacial Maximum. Chigaku-Zusshi (Journal of Geography, Japan), 103: 867-885.

Frey, H., Machguth, H., Huss, M., Huggel, C., Bajracharya, S., Bolch, T., Kulkarni, A., Linsbauer, A., Salzmann, N., \& Stoffel, M. (2014). Estimating the volume of glaciers in the Himalayan-Karakoram region using different methods. The Cryosphere, 8(6):2313-2333. doi: 10.5194/tc-8-2313-2014.

Furbish, D.J. \& Andrews, J.T. (1984). The use of hypsometry to indicate long-term stability and response of valley glaciers to changes in mass transfer. Journal of Glaciology 30, 199-211

Gantayat, P., Kulkarni, A., \& Srinivasan, J. (2014). Estimation of ice thickness using surface velocities and slope: case study at Gangotri Glacier, India. Journal of Glaciology, 60(220):277-282. doi: 10.3189/2014JoG13J078.

Geikie, J. (1909). The Alps during the glacial peeiod. Scottish Geographical Magazine, 25(9), 481491. https://doi.org/10.1080/00369220908733983

Glen, J. W. (1955). The creep of polycrystalline ice. Proceedings of the Royal Society of London, Series A, 228(1175):519-538. 
Gross, G., Kerschner, H. \& Patzelt, G. (1977). Methodische Untersuchungen über die Schneegrenze in alpine Gletschergebieten. Zeitschr. für Gletscherkunde und Glazialgeologie, XII (2): 223-251.

Haeberli, W. \& Hoelzle, M. (1995). Application of inventory data for estimating characteristics of and regional climate-change effects on mountain glaciers: a pilot study with the European Alps, Ann. Glaciol., 21, 206-212, doi:10.3198/1995AoG21-1-206-212.

Hawkins, F. F. (1980). "Glacial Geology and Late Quaternary Paleoenvironment in the Merchants Bay Area, Baffin Island, N.W.T., Canada." M.A. thesis, University of Colorado.

Hoinkes, H. (1970). Methoden und Möglichkeiten von Massenhaushaltsstudien auf Gletschern. Zeitschrift für Gletscherkunde und Glazialgeologie, 6-2: 37-90, Innsbruck.

Hubbard, B. \& Glasser, N. (2005). Field Techniques in Glaciology and Glacial Geomorphology. Wiley, Chichester. 400p.

Huss, M. (2012) Extrapolating glacier mass balance to the mountain range scale: the European Alps 1900-2100. Cryosphere, 6(4), 713-727 (doi: 10.5194/tc-6-713-2012)

Huss, M. \& Farinotti, D. (2012). Distributed ice thickness and volume of all glaciers around the globe. Journal of Geophysical Research, 117:F04010. doi: 10.1029/2012JF002523.

IGUL (1996). Légende pour le levé de cartes géomorphologiques au 1:10.000, Institut de Géographie. Université de Lausanne., 3ème édition ed., 13 pp.

James, W. \& Carrivick, J. (2016). Automated modelling of spatially-distributed glacier ice thickness and volume. Computers \& Geosciences. 92. 10.1016/j.cageo.2016.04.007.

Jiskoot, H. (2011). Dynamics of Glaciers. Encyclopedia of snow, ice and glaciers. In: Encyclopedia of snow, ice and glaciers. p. 245-256.

Kaser, G. \& Kerschner, H. (1994). Equilibrium line altitudes of Younger Dryas glaciers in the Alps as paleoclimatic information sources. Annales Geophysicae, 12-2: 386.

Kaser, G., Fontain, A., \& Jansson, P. (2003). A manual for monitoring the mass balance of mountain glaciers. A contribution from the International Commission on Snow and Ice (ICSI) to the UNESCO HKH-Friend program.

Kaser, G., Cogley, JG., Dyurgerov, M. B., Meier, M. \& Ohmura, A. (2006) Mass balance of glaciers and ice caps: consensus estimates for 1961-2004. Geophys. Res. Lett., 33(19), L19501 (doi: 10.1029/2006GLO27511)

Kessler, M.A., Anderson, R.S., \& Stock, G.M. (2006). Modeling topographic and climatic control of east-west asymmetry in sierra nevada glacier length during the last glacial maximum. Journal of Geophysical Research: Earth Surface, 111(F2), F02002. doi: 10.1029/2005JF000365 
Klein, A. G., Seltzer, G. O. \& Isacks, B. L. (1999). Modern and Last Local Glacial Maximum snowlines in the Central Andes of Peru, Bolivia, and Northern Chile. Quaternary Research Reviews, 18: 3-84.

Kurowski, L. (1891). Die Höhe der Schneegrenze. Penck's Geographische Abhanddlungen, 51 (124): 119-160.

Lambiel, C., Maillard, B., Regamei, B., Martin, S., Kummert, M., Schoeneich, P., Pellitero Ondicol, R. \& Reynard, E. (2013). Adaptation of the geomorphological mapping system of the University of Lausanne for ArcGIS. In 8th International Conference on Geomorphology (IAG), Paris, 2731 Aug. 2013.

Lichtenecker, N. (1936). Die gegenw\&auml;rtige und die eiszeitliche Schnegrenze in den Ostalpen. Verhandlungen der III Internationalen Quartarkonferenz, Wien, 141-47.

Linsbauer, A., Paul, F., Hoelzle, M., Frey, H., and Haeberli, W. (2009). The Swiss Alps without glaciers - A GIS-based modelling approach for reconstruction of glacier beds, in: Proceedings of Geomorphometry 2009, Zurich, 243-247.

Linsbauer, A., Paul, F., \& Haeberli, W. (2012). Modeling glacier thickness distribution and bed topography over entire mountain ranges with GlabTop: Application of a fast and robust approach. Journal of Geophysical Research, 117:F03007. doi: 10.1029/2011JF002313.

López-Moreno, I., Revuelto, J., Rico, I., Chueca-Cía, J., Julián, A.,Serreta, A., Serrano, E., Vicente-Serrano, S., Azorin-Molina, C., Alonso-González, E., \& García-Ruiz, J. M. (2016). Thinning of the Monte Perdido Glacier in the Spanish Pyrenees since 1981. The Cryosphere. 10. 681-694. 10.5194/ tc-10-681-2016.

Louis, H. (1955). Schneegrenze und Schneegrenzbestimmung. Geographisches Taschenbuch, 1954/55: 414-418.

Lukas, S. (2007). Early-Holocene glacier fluctuations in Krundalen, south central Norway: palaeo-glacier dynamics and palaeoclimate. Holocene 17, 585-598.

Mackintosh, A. N. Anderson, B. M. \& Pierrehumbert, R. T. (2017). Reconstructing climate from glaciers. Annual Review of Earth and Planetary Sciences, 45, 649-680. https://doi.org/10.1146/ annurev-earth-063016-020643

Mahaney, W. C. (1990). Ice on the Equator: Quaternary Geology of Mount Kenya, East Africa. Wm Caxton Ltd., Sister Bay, WI 386pp.

Meierding, T. C. (1982). Late Pleistocene glacial equilibrium-line altitudes in the Colorado Front Range: a comparison of methods, Quater- nary Res., 18, 289-310.

Meier, M. F. \& Post, A. S. (1962). Recent variations in mass net budgets of glaciers in western North America. En: Comm. des Neiges et des Glaces. Union Geodesique et Geophysique Internationale. Assoc. Internat. d'Hydr. Scient, pp 63-77, Oberqurgl. 
Mercer, J. H. (1961). The response of fjord glacies to changes in the firn limit. Journal of Glaciology $3,850-858$

Miller, G. H., Bradley, R. S., \& Andrews, J. T. (1975). The glaciation level and lowest equilibrium line altitude in the high Canadian Arctic: maps and climatic interpretation. Arctic and Alpine Research 7, 155- 168.

Nesje, A. \& Dahl, S. O. (2000). Glaciers and Environmental Change, Arnold, London, 2000, pp. 203

Nogami, M. (1972). The snow line and climate during the last glacial period in the Andes mountains. Daiyonki-Kenkyu (The Quaternary Research) 11, 71-80 (in Japanese).

Nogami, M. (1976). Altitude of the modern snowline and Pleistocene snowline in the Andes. Geographical Reports of Tokyo Metropoli- tan University 11, 71-86.

Nye, J. F. (1952). The mechanics of glacier flow. J Glacialogy. 2:82-93.

Nye, J. F. (1965). The flow of a glacier in a channel of rectangular, elliptic or parabolic cross-section. Journal of glaciology. p. 661-690.

Osmaston, H. (1965). The Past and Present Climate and vegetation of Rwenzori Glaciers. Uganda Journal, 25: 99-104.

Osmaston, H. (1975). Models for the estimation of firnlines of present and Pleistocene glaciers. En: R. F. Peel, M.D.I. Chisholm and P. Hagget (Editors), Processes in Physical and Human Geography. 218-245. Bristol Essays, Bristol (UK).

Osmaston, H. A. (1989a). Glaciers, Glaciations and Equilibrium Line Altitudes on the Ruwenzori. Quaternary and Environmental Research on East African Mountains. Brookfield, Balkema, Rotterdam. p. 31-104.

Osmaston, H. (1989b). Glaciers, glaciations and equilibrium line altitudes on Kilimanjaro. In: Mahaney, W.C. (Ed.), Quaternary and Environmental Research on East African Mountains. Balkema, Rotterdam, A.A, pp. 7-30.

Osmaston, H. A. (2005). Estimates of glacier equilibrium line altitudes by the AreaxAltitude, the AreaxAltitude Balance Ratio and the AreaxAltitude Balance Index methods and their validation. Quaternary International, 138-139: 22-31. http://dx.doi.org/10.1016/j.quaint.2005.02.004

Østrem, G. (1966). The height of the glaciation limit in southern British Columbia and Alberta. Geografiska Annaler 48A, 126-138

Østrem, G., \& Stanley, A. (1966). Glacier Mass-balance Measurements: A Manual for Field Work, Department of Mines and Technical Surveys, Ottawa. 81p. 
Østrem, G., and Stanley, A. (1969). Glacier Mass-balance Measurements: A Manual for Field and Office Work, Energy, Mines and Resources Canada, Ottawa, and Norwegian Water Resources and Electricity Board, Oslo. 129p.

Østrem, G., \& Brugman, M. (1991). Glacier Mass-Balance Measurements: A manual for field and office work, NHRI Science Report 4, Norwegian Water Resources and Energy and Energy Administration/Environment Canada, Saskatoon, Canada.

Palma, P., Oliva, M., García-Hernández, C., Gómez Ortiz, A., Ruiz-Fernández, J., Salvador-Franch, F. \& Catarineu, M. (2017). Spatial characterization of glacial and periglacial landforms in the highlands of Sierra Nevada (Spain). Sci Total Environ. 584-585:1256-1267. doi:10.1016/j.scitotenv.2017.01.196.

Pellitero, R., Rea, B. R., Spagnolo, M., Bakke, J., Ivy-Ochs, S., Frew, C. R., Hughes, P., Ribolini, A., Lukas, S. \& Renssen, H. (2016). GlaRe, a GIS tool to reconstruct the 3D surface of palaeoglaciers. Comput Geosci. 94:77-85. doi:10.1016/j.cageo.2016.06.008.

Penck, A. (1906). Climatic features of the pleistocene ice age. The Geographical Journal, 27(2), 182-187. https://doi.org/10.2307/1776673

Peña, J., Pellicer, F., Chueca, J., \& Julián, A. (1997). Leyenda para mapas geomorfológicos a escalas 1:25.000/1:50.000. In J. L. Peña (Ed.), Cartografía Geomorfológica Básica y Aplicada. Logroño: Geoforma Ed.

Péwé, T. L. \& Reger, R. D. (1972). Modern and wisconsinan snowlines in Alaska. En: Procceding of the XXIV International Geological Congress, secc12, pp. 187-197.

Porter, S. C. (1975a). Equilibrium-line altitudes of late Quaternary glaciers in the Southern Alps, New Zealand, Quaternary Res., 5, 27-47.

Porter, S. C. (1975b). Glaciation limit in New Zealand's Southern Alps. Arctic and Alpine Research 7, 33-37.

Porter, S. C. (1977). Present and past glaciation threshold in the Cascade Range, Washington, U.S.A.: topographic and climatic controls, and paleoclimatic implications. Journal of Glaciology $18,101-116$.

Porter, S. C. (1979). Hawaiian glacial ages. Quaternary Research 12, 161-187.

Porter, S. C. (1981). Glaciological evidence of Holocene climatic change. In: Wigley, T.M.L., Ingram, M.J., Farmer, G. (Eds.), Climate and History. Cambridge University Press, Cambridge, pp. 82-110.

Porter, S. C., Pierce, K. L. \& Hamilton, T. D. (1983). Late Wisconsin moun- tain glaciation in the western United States. In: Porter, S.C. (Ed.), Late Quaternary Environments of the United States: the Late Pleistocene. University of Minnesota Press, Minneapolis, pp. 71-111. 
Porter, S. C. (2001). Snowline depression in the tropics during the last glaciation. Quaternary Science Review, 20: 1067-1091.

Ramage, J. M., Smith, J. A., Rodbell, D. T. \& Seltzer, G. O. (2005). Comparing reconstructed Pleistocene equilibrium-line altitudes in the tropical Andes of central Peru. Journal of Quaternary Science, 20: 777-788.

Rea, B. R. \& Evans, D. J. A. (2007). Quantifying climate and glacier mass balance in North Norway during the Younger Dryas. Palaeogeography, Palaeoclimatology, Palaeoecology 246, 307-330.

Rea, B. R. (2009). Defining modern day area-altitude balance ratios (AABRs) and their use in glacier-climate reconstructions. Quat. Sci. Rev. 28 (3-4), 237-248.

Richter, E. (1885). Beobachtungen an den Gletschern der Ostalpen. II. Die Gletscher der Ötzhaler Gruppe im Jahre 1883. Zeitschrift des Deutschen und Österreichischen Alpenvereins 16: 54-65, Innsbruck.

Richter, E. (1888). Die Gletscher der Ostalpen. J. Engelhon, Stuttgart.

Rivera, A., Bown, F., Napoleoni, F., Muñoz, C. \& Vuille, M. (2017). Manual Balance de Masa Glaciar. Ediciones CECs, Valdivia, Chile.

Rodbell, D. T. 1992. Late Pleistocene equilibrium-line reconstructions in the northern Peruvian Andes. Boreas, 21: 43-52.

Schoeneich, P. (1993). Comparaison des systèmes de légendes français, allemand et Suisse. Principes de la légende IGUL. In Schoeneich P., Reynard E. (eds). Cartographie géomorphologiques, cartographie des risques . Lausanne, Institut de Géographie, Travaux et Recherches, 9, (p. 15 24).

Schilling, D. H. \& Hollin J. (1981). Numerical reconstructions of valley glaciers and small ice caps. In: Denton GH, Hughes TJ. (Eds.), The Last Great Ice Sheets. New York: Wiley, pp. 207-220.

Serrano, E. \& González Trueba, J. J. (2004). El método A.A.R. para la determinación de paleo-ELAs: Análisis metodológico y aplicación en el Macizo de Valdecebollas (Cordillera Cantábrica). Cuadernos de Investigación Geográfica, 30: 7-32.

Sissons, J. B. (1974). A late glacial ice cap in the central Grampi- ans, Scotland. Transactions of the Institute of British Geog- raphers, 62: 95-114. http://dx.doi.org/10.2307/621517

Sissons, J. B. (1980). The Loch Lomond advance in the Lake Dis- trict, northern England. Transactions Royal Society Edin- burgh: Earth Sciences, 71: 13-27. http://dx.doi.org/10.1017/ S0263593300013468

Six, D., \& Vincent, C. (2014). Sensitivity of mass balance and equilibrium-line altitude to climate change in the French Alps. Journal of Glaciology, 60(223), 867-878. doi:10.3189/2014JoG14J014 
Úbeda, J., Alcalá, J. \& Palacios, D. (2006). Máximo avance glaciar y fases de deglaciación del complejo volcánico Nevado Coropuna (Arequipa-Perú): estado actual de la investigación. En: S.G.d. Perú (Editor), XIII Congreso Peruano de Geología, Lima (Perú).

Úbeda, J. (2007). Caracterización Geomorfológica del sector septentrional de la Zona Volcánica Central de los Andes Centrales. Planteamiento de un caso de estudio: el sistema glaciar del complejo volcánico Nevado Coropuna. Trabajo de investigación para la obtención del Diploma de Estudios Avanzados, Universidad Complutense de Madrid, Madrid, 312 pp.

Úbeda, J., (2011). El impacto del cambio climático en los glaciares del complejo volcánico Nevado Coropuna (Cordillera Occidental de los Andes Centrales). Tesis doctoral. Universidad Complutense de Madrid. http://eprints.ucm.es/12076/

Van der Veen, C. J., (1999). Fundamentals of Glacier Dynamics. Rotterdam: Balkema.

Vieira, G. (2008). Combined numerical and geomorphological reconstruction of the Serra da Estrela plateau icefield, Portugal. Geomorphology. 97:190-207. doi:10.1016/j.geomorph.2007.02.042.

Visser, P. C. (1938). Wissenschaftliche Ergebnisse der Niederlandischen Expeditionen in den Karakorum und die angrenzenden Gebiete in Den Jahren 1922-1935. Band II: Glaziologie. Leiden: Brill.

Wahrhaftig, C. \& Birman, J. (1965). The Quaternary of the Pacific Mountain system in California. In "The Quaternary of the United States" (H. E. Wright, Jr., and D. G. Frey, Eds.), pp. 299-340. Princeton, Univ. Press, Princeton, N.J.

Weidick, A. (1968). Observations on some Holocene glacier fluctuations in West Greenland. Meddeleser om Grønland 165(6), 0- 194.

WGMS website. (2018). https://wgms.ch

White, S. E. (1981). Equilibrium line altitudes of late Pleistocene and recent glaciers in central Mexico. Geografiska Annaler 63, 241-249.

White, S. E. \& Valastro Jr., S. (1984). Pleistocene glaciation of Volcano Ajusco, central Mexico, and comparison with the standard Mexican glacial sequence. Quaternary Research 21, 21-35. 